\title{
Exceptional AGN long-timescale X-ray variability: The case of PHL 1092
}

\author{
G. Miniutti ${ }^{1, a}$, W.N. Brandt²,3, D.P. Schneider ${ }^{2,3}$, A.C. Fabian ${ }^{4}$, L.C. Gallo ${ }^{5}$, \\ and Th. Boller ${ }^{6}$ \\ ${ }^{1}$ Centro de Astrobiología (CSIC-INTA), Dep. de Astrofísica; ESA, PO Box 78, \\ 28691 Villanueva de la Cañada, Madrid \\ ${ }^{2}$ Department of Astronomy and Astrophysics, The Pennsylvania State University, 525 Davey Lab., \\ Univeristy Park, PA 16802, USA \\ ${ }^{3}$ Institute for Gravitation and the Cosmos, The Pennsylvania State University, University Park, PA 16802, USA \\ ${ }^{4}$ Institute of Astronomy, Madingley Road, Cambridge CB3 OHA, UK \\ ${ }^{5}$ Department of Astronomy \& Physics, Saint Mary's University, 923 Robie Street, Halifax, NS B3H 3C3, UK \\ ${ }^{6}$ Max-Planck-Institut für extraterrestrische Physik, Postfach 1312, 85741 Garching, Germany
}

\begin{abstract}
PHL 1092 is a $z \sim 0.4$ high-luminosity counterpart of the class of Narrow-Line Seyfert 1 galaxies. In 2008, PHL 1092 was found to be in a remarkably low X-ray flux state during an XMM-Newton observation. Its $2 \mathrm{keV}$ flux density had dropped by a factor of $\sim 260$ with respect to a previous observation performed $4.5 \mathrm{yr}$ earlier. The UV flux remained almost constant, resulting in a significant steepening of the optical-to-X-ray slope $\alpha_{\mathrm{ox}}$ from -1.57 to -2.51 , making PHL 1092 one of the most extreme X-ray weak quasars with no observed broad absorption lines (BALs) in the UV. We have monitored the source since 2008 with three further XMM-Newton observations, producing a simultaneous UV and X-ray database spanning almost $10 \mathrm{yr}$ in total in the activity of the source. We present here results from our monitoring campaign.
\end{abstract}

\section{INTRODUCTION}

A growing number of X-ray weak quasars do not show any detectable broad absorption lines (BALs) in their UV spectra (e.g. Wu et al. 2011). The best-studied case is that of PHL 1811 (Leighly et al. 2001, 2007a, 2007b). PHL $1092(z=0.396)$ is a high-luminosity Narrow-Line Seyfert 1 (NLS1) galaxy with broad emission line FWHM $\sim 1800 \mathrm{~km} \mathrm{~s}^{-1}$, [O III] $\lambda 5007 / \mathrm{H} \beta \sim 0.9$, and outstanding Fe II emission $(\mathrm{Fe} \mathrm{II} / \mathrm{H} \beta=1.81)$. Its $\mathrm{UV}$ spectrum does not show any sign of absorption and it exhibits relatively weak, broad, and blueshifted C IV emission and presents many analogies with PHL 1811. In 2008, PHL 1092 was found to be in a remarkably low X-ray flux state during an $X M M-N e w t o n$ observation with a $2 \mathrm{keV}$ flux-density drop of $\sim 260$ with respect to a previous 2003 XMM-Newton exposure (Miniutti et al. 2009). The UV flux remained almost constant, resulting in a significant steepening of the optical-to-X-ray slope $\alpha_{\text {ox }}$ from -1.57 to -2.51 and showing that even extreme X-ray weakness can in fact be a transient phenomenon.

We report here results from an X-ray monitoring campaign spanning almost $10 \mathrm{yr}$ in total in the activity of the source. Our observations provide simultaneous UV and $\mathrm{X}$-ray data with which we follow the long-term X-ray and optical/UV variability of this remarkable source. A more completed analysis can be found in Miniutti et al. (2012).

\section{EXTREME LONG-TERM X-RAY VARIABILITY}

The historical soft X-ray flux light curve of PHL 1092 is shown in Fig. 1 (upper panel). As can be seen, PHL 1092

\footnotetext{
a e-mail: gminiutti@cab.inta-csic.es
}

was observed in an extremely low flux state in 2008, which was reported and discussed by Miniutti et al. (2009). Its soft X-ray flux then recovered to reach in 2010 almost the same flux level it had in 2000. The XMMNewton observations provide simultaneous photometric data in the UV via the Optical Monitor (OM). The presence of simultaneous UV and X-ray data allows us to derive reliably the optical-to-X-ray slope $\alpha_{\mathrm{ox}}$ from 2000 to 2010. Here we use the standard definition of $\alpha_{\text {ox }}=\log \left(f_{2} / f_{2500}\right) \log \left(v_{2} / v_{2500}\right)^{-1}=0.384 \log \left(f_{2} / f_{2500}\right)$ between $2 \mathrm{keV}$ and $2500 \AA$ in the rest-frame. Following Gibson, Brandt $\&$ Schneider (2008), we also define $\Delta \alpha_{\text {ox }}$ as the difference between the observed $\alpha_{\mathrm{ox}}$ and that expected from the anti-correlation between $\alpha_{\text {ox }}$ and the $2500 \AA$ monochromatic luminosity which implies $\alpha_{\mathrm{ox}}^{\text {expected }}=$ -1.48 for PHL 1092 (e.g. Just et al. 2007). In Fig. 1 (lower panel) we show $\Delta \alpha_{\text {ox }}$ as a function of the $2 \mathrm{keV}$ monochromatic flux. The linear correlation with $\log f_{2 \mathrm{keV}}$ shows that the $\alpha_{\mathrm{ox}}$ variability of PHL 1092 can be entirely explained in terms of its long-term X-ray flux changes. The UV flux is almost constant (within 10\% of its mean value), indicating that the large $\alpha_{\mathrm{ox}}$ variability of PHL 1092 has little to do with mass accretion rate variations, and is instead related to physical processes affecting the X-ray part of the SED only.

\section{X-RAY SPECTRA}

No X-ray spectral information is available for XMM(1), so we perform our analysis considering observations $\mathrm{XMM}(2)$ to $\mathrm{XMM}(6)$ spanning $7.5 \mathrm{yr}$ (5.4 yr in the restframe) in the activity of PHL 1092 and more than two orders of magnitude in soft X-ray flux. All EPIC-pn data are considered between $0.3 \mathrm{keV}$ and up to the energy 

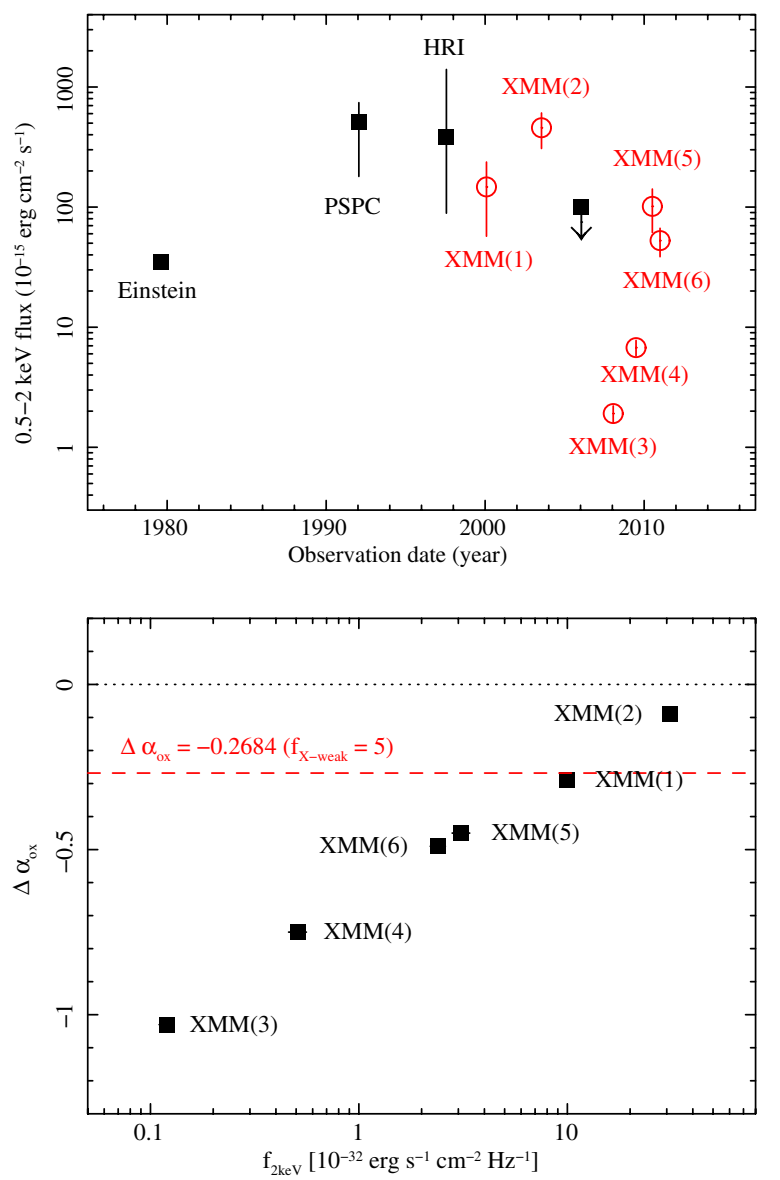

Figure 1. Upper: the historical $0.5-2 \mathrm{keV}$ X-ray flux of PHL 1092. The data points represent the mean flux level during the observation, and the error is a measure of the intraobservation variability rather than of the statistical error on the mean flux. The upper limit is from an XMM-Newton slew in 2006. Lower: the 2000-2010 $\Delta \alpha_{\text {ox }}$ of PHL 1092 as a function of its $2 \mathrm{keV}$ flux density. The level $\Delta \alpha_{\mathrm{ox}}=0$ corresponds to $\alpha_{\mathrm{ox}}=-1.48$, which is that expected for a quasar with the $2500 \AA$ monochromatic luminosity of PHL 1092. PHL 1092 was X-ray weak by a factor $f_{X \text {-weak }} \simeq 480$ during $\operatorname{XMM}(3)$.

at which the source is confidently detected above the background. The EPIC-pn spectra used in our analysis are shown in Fig. 2.

We adopt as our baseline model the recently developed OPTXAGN model (see Done et al. 2012; Jin et al. 2012 for a detailed description and applications). This model assumes that the disc emits, at each radius, a colour-temperature corrected blackbody down to a given $\mathrm{X}$-ray corona outer radius $\mathrm{R}_{\mathrm{c}}$. The main model assumption is that the energy can no longer thermalise within $R_{\mathrm{c}}$, and is instead distributed between powering the soft $\mathrm{X}$-ray excess component and the high energy powerlaw tail via Comptonization in a two-phase plasma. The energy distribution within the corona is determined by the fraction of coronal luminosity powering the highenergy power-law $f_{\mathrm{pl}}$ (the remaining coronal luminosity $1-f_{\mathrm{pl}}$ powers the soft excess). As mentioned, $R_{\mathrm{c}}$ sets the X-ray corona outer radius, while its inner boundary coincides with the innermost stable circular orbit (ISCO) $R_{\text {isco. The total coronal luminosity is proportional to }}$

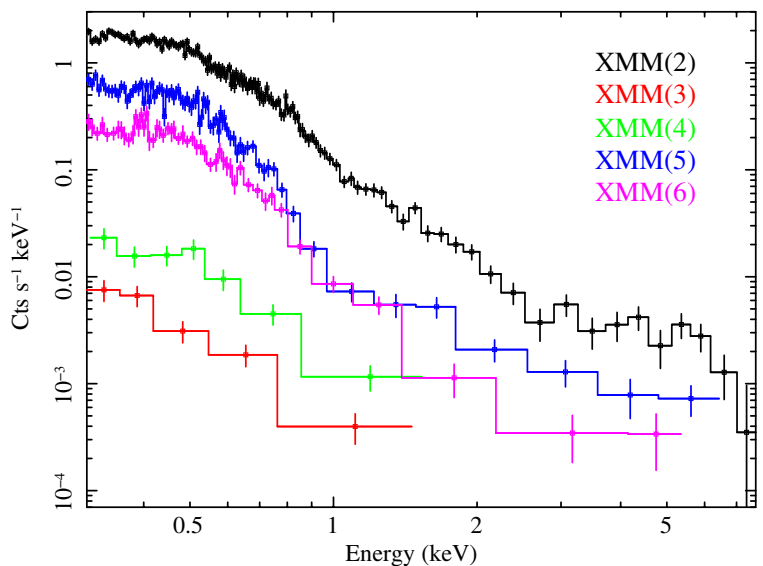

Figure 2. The EPIC-pn spectra of PHL 1092 used in our analysis from 2003 to 2010. All data are considered down to $0.3 \mathrm{keV}$ and up to the observation-dependent energy at which the source is confidently detected above the background level. Observation $\mathrm{XMM}(1)$ is not considered here as no spectral data are available.

$1-R_{\text {isco }} / R_{\mathrm{c}}$ so that no $\mathrm{X}$-ray emission is produced if $R_{\mathrm{c}} \leq R_{\text {isco }}$

\subsection{An absorption-driven interpretation}

We first look for a solution in which the intrinsic X-ray flux of PHL 1092 is constant and possibly similar to that expected for a quasar with its optical luminosity. Guided by the observational fact that X-ray weak quasars are often associated with intrinsic UV and X-ray absorption (at least the BAL X-ray weak sources), we assume that the X-ray flux variability of PHL 1092 is apparent and due to X-ray absorption by ionized gas partially covering the X-ray source. After a few initial tests, we found that the absorber column density and covering fraction cannot be simultaneously constrained. We then decided to force a marginally Compton-thick column density of $\mathrm{N}_{\mathrm{H}}=$ $10^{24} \mathrm{~cm}^{-2}$ in all observations and fit for the covering fraction and ionization state.

The model leaves significant residuals in the intermediate flux observations $\mathrm{XMM}(5)$ and $\mathrm{XMM}(6)$, which appear to exhibit a further absorption structure at $\sim 1 \mathrm{keV}$ $(\sim 1.4 \mathrm{keV}$ in the rest-frame). To address this feature, we added a Gaussian absorption line with the same energy and width in all observations, but we allow the absorption line optical depth to be observation-dependent. We obtain a good description of the data $\left(\chi^{2} /\right.$ dof $\left.=410 / 355\right)$ with a relatively broad absorption line $\left(\sigma_{\mathrm{abs}} \sim 0.20 \mathrm{keV}\right)$ at $E_{\text {abs }} \sim 1.05 \mathrm{keV}$ in the observed-frame, corresponding to $\sim 1.47 \mathrm{keV}$ in the rest-frame. The line is more prominent in $\mathrm{XMM}(5)$ and $\mathrm{XMM}(6)$ with optical depths of $\tau_{(5)}=1.00 \pm$ 0.10 and $\tau_{(6)}=0.60 \pm 0.10$. The broad width and energy of the absorption line suggests an identification with the Fe $\mathrm{L}$ absorption complex around $1 \mathrm{keV}$, with possible contribution from $\mathrm{Ne}$ IX-X and/or Mg XI-XII. Identification of the absorption feature with $\mathrm{Fe} \mathrm{L}$ resonant absorption at $\sim 1 \mathrm{keV}$ implies an outflow with highly relativistic velocity $v_{\text {out }} \sim 0.3 \mathrm{c}$.

On the other hand, the X-ray flux variability is produced by the variation of the covering fraction 

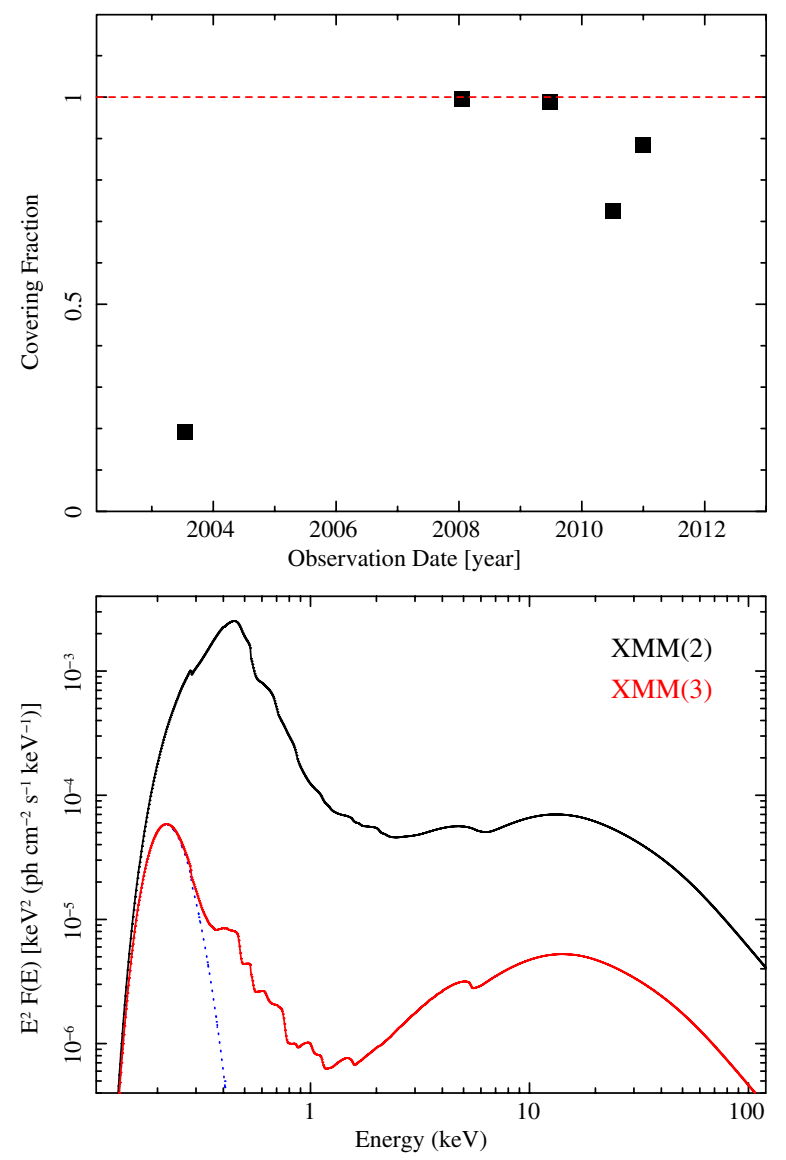

Figure 3. Upper: the evolution of the absorber covering fraction with time. The lowest flux states observed in 2008 and 2009 are due to the $\sim 100 \%$ covering of the X-ray source. Lower: the $\mathrm{E}^{2} \mathrm{~F}(\mathrm{E})$ model X-ray SED of PHL 1092 during the highest and lowest flux observations $\mathrm{XMM}(2)$ and $\mathrm{XMM}(3)$ as obtained with the disc reflection-dominated model. The SED has been corrected for the intrinsic warm absorption, while Galactic absorption is present. The X-ray SED is completely reflection-dominated with some accretion disc contribution at soft X-rays (blue dashed line) which becomes visible in the low X-ray flux observations.

of a relatively cold $(\log \xi \leq-1.0)$ absorber with $\mathrm{N}_{\mathrm{H}}=$ $10^{24} \mathrm{~cm}^{-2}$ (fixed, as discussed above). The absorber covers $\sim 19 \%$ of the X-ray source in the highest X-ray flux observation, and $\sim 99.6 \%$ of it during the lowest X-ray flux one. The evolution of the cold absorber covering fraction is shown in Fig. 3 (upper panel). Remarkably, if the absorber were absent, the intrinsic monochromatic flux of PHL 1092 at $2 \mathrm{keV}$ would make it a standard quasar with constant $\Delta \alpha_{\text {ox }}=-0.05 \simeq 0$. Thus, within the context of the absorption model, PHL 1092 would be a quasar with standard X-ray output, which only appears extremely $\mathrm{X}$-ray weak at times due to intervening absorption. We measure a corona size of $15 \pm 5 r_{g}$, which mostly powers the soft X-ray excess, only 5 per cent of the available energy being associated with the high energy power law.

\subsection{A disc reflection interpretation}

In the absorption-driven model discussed above, the soft excess is due to Compton up-scattering of soft UV/EUV disc photons in an optically thick corona with plasma temperature $\sim 150 \mathrm{eV}$. This interpretation of the soft excess has been freqeuntly questioned by many different authors (e.g. Gierliński \& Done 2004) because, in order to reproduce the uniform spectral shape in samples of AGN with significantly different $\mathrm{BH}$ mass and Eddington ratio, the electron temperature must be remarkably uniform. The uniform soft excess shape may indicate that processes that are independent of accretion physics are instead at play. One appealing possibility is that ionised reflection off the inner accretion disc contributes to the soft X-rays. X-ray reflection off the inner accretion disc has been shown to represent a plausible explanation of the nature of the soft excess in many sources and, in particular, in many NLS1 galaxies (e.g. 1H 0707-495, Fabian et al. 2004, 2009; NGC 4051, Ponti et al. 2006; IRAS 13224-3809, Ponti et al. 2010).

We apply the baseline OPTXAGN model as above to all EPIC data, but we force the X-ray corona to only power the hard X-ray power-law $\left(f_{\mathrm{pl}}=1\right)$ so that the baseline model does not produce any soft excess (the corona is optically thin only). To describe the soft X-ray excess as ionised disc reflection, we use the the reflection model of Ross $\&$ Fabian (2005) to which we apply a relativistic kernel, appropriate for a Kerr $\mathrm{BH}$. As the corona is now optically thin, the inner disc below $R_{\mathrm{c}}$ is visible and we fix the inner disc radius to $R_{\text {isco }} \simeq 1.24 r_{g}$ at all flux levels.

Our initial fits show that the X-ray spectrum of PHL 1092 is completely reflection-dominated at all flux levels. We thus force the corona outer radius $\mathrm{R}_{\mathrm{c}}$ to coincide with $\mathrm{R}_{\mathrm{isco}}$ so that no $\mathrm{X}$-ray corona emission is present. We obtain a fair description of the data $\left(\chi^{2} /\right.$ dof $\left.=420 / 353\right)$ with the inclusion of a low-column/low-ionisation warm absorber.

One key result of this model is shown in the bottom panel of Fig. 3 (lower panel), where we display the model $\mathrm{X}$-ray SED for the highest and lowest flux states. The key point is that the actual reflection flux change is only a factor $\sim 10$ between the two flux states and not the factor $\sim 260$ observed at $2 \mathrm{keV}$. This aspect is best seen above $\sim 30 \mathrm{keV}$ where ionisation effects are negligible. The flux change is larger than two orders of magnitude at soft X-rays, mostly because of the different disc ionisation.

The other key result is that the X-ray spectrum appears reflection-dominated at all flux levels. This is understood in terms of a very compact X-ray corona located in the immediate vicinity of the black hole event horizon. In this situation, light-bending naturally effects produce reflection-dominated spectra in which the primary powerlaw-like emission is strongly bent towards the disc and balck hole, with only a small fraction being able to escape to a distant observer (Miniutti et al. 2003: Miniutti \& Fabian 2004).

It turns out that the total (continuum + reflection) flux density at $2 \mathrm{keV}$ in the absence of light-bending effects is higher than the observed (reflection only) by a factor $\sim 70$ during the low flux state and by a factor $\sim 9$ during the high flux one. This result implies that PHL 1092 would be a quasar with relatively standard X-ray output and $\Delta \alpha_{\mathrm{ox}} \sim 0$ oscillating between X-ray weak and X-ray bright states in the absence of light-bending effects (i.e. if it had a less compact and centrally concentrated X-ray corona). 


\section{CONCLUSIONS}

We have analysed XMM-Newton observations of PHL 1092 covering nearly $10 \mathrm{yr}(7.5 \mathrm{yr}$ in the rest-frame) of its activity and spanning more than two orders of magnitude in soft X-ray flux. The main results of our analysis can be summarised as follows (see Miniutti et al. 2012 for a more complete discussion):

1. The remarkable $\alpha_{\mathrm{ox}}$ variability of PHL 1092 is entirely driven by the X-ray variability. During our monitoring campaign, we detect a maximum X-ray flux variability by a factor $\sim 260$ in $\sim 3.5 \mathrm{yr}$ (rest-frame), while the UV variability is confined to within $10-15 \%$. At its minimum X-ray flux level (January 2008), PHL 1092 is about a factor of $\sim 480$ $\mathrm{X}$-ray weak with respect to a standard quasar with its optical luminosity.

2. We propose here two possible scenarios that can explain the UV-to-X-ray SED of PHL 1092 at all X-ray flux levels: i) an absorption model where the X-ray variability is dominated by changes of the covering fraction of a relatively cold absorber which covers almost $100 \%$ of the X-ray source in $\mathrm{X}$-ray weak states; ii) a disc-reflection-dominated scenario where the X-ray corona is confined within few $r_{g}$ from the central BH at all flux levels. In this case the source intrinsic variability only needs to be of a factor of $\sim 10$ rather than the observed factor of $\sim 260$.

The absorption interpretation is not completely selfconsistent as the X-ray flux variability is produced by variations of a relatively cold absorber. The absorber should imprint some absorption feature in the UV, but UV absorption is not detected in the HST spectrum of PHL 1092. One possibility is that the absorber is confined within the UV-emitting disc, i.e. within a few tens of $r_{g}$. If so, however, absorbing clouds cannot survive at the low ionisation level we need to reproduce the X-ray variability. A possible way out would be to consider a multi-layer absorption model in which every absorbing cloud is structured into multiple layers. The outer layers may protect the innermost cloud(s) nucleus from the ionising flux, thus enabling them to survive close to the source at low ionisation. We defer such study to future work.

As mentioned, the X-ray variability and overall SED can also be reproduced in a reflection-dominated scenario where a compact X-ray corona is confined within few $r_{g}$ from the $\mathrm{BH}$ at all flux levels. Light-bending effects produce a disc reflection-dominated spectrum, and the observed extreme X-ray flux variability at soft X-rays (a factor 260 in $3.5 \mathrm{yr}$ ) is reproduced with a much smaller factor of $\sim 10$ intrinsic variation. A similar scenario has been invoked for 1H 0707-495 and IRAS 13224-3809, two NLS 1 galaxies which share many properties with PHL 1092 from UV to X-rays. The recent detection of soft X-ray time lags in 1H 0707-495 and IRAS 13224-3809 (Fabian et al. 2009; 2012) supports a reflection scenario in these objects.

The two scenarios we suggest make very different predictions for the X-ray flux above $10 \mathrm{keV}$. The absorption model implies a constant flux $\sim 2.5 \times 10^{-13} \mathrm{erg} \mathrm{cm}^{-2} \mathrm{~s}^{-1}$ in the $30-100 \mathrm{keV}$ band, while the reflection-dominated model predicts a much lower flux of $\sim 4.1 \times 10^{-14} \mathrm{erg}$ $\mathrm{cm}^{-2} \mathrm{~s}^{-1}$. Deep observations of PHL 1092 (or of a similar source) with sensitive hard X-ray detectors such as $A S T R O-H$ and $N U S T A R$ in the near future may thus reveal the nature of the transient X-ray weakness phenomenon, and could shed light into the nature of the non-BAL X-ray weak quasars population at large.

Based on observations obtained with XMM-Newton, an ESA science mission with instruments and contributions directly funded by ESA Member States and NASA. GM thanks the Spanish Ministerio de Ciencia e Innovación and CSIC for support through a Ramón y Cajal contract. Financial support for this work was also provided by the Spanish Ministry of Science and Innovation through grant AYA2010-21490-C02-02. GM also thanks CSIC for the award of grant PA1003039, as well as the IoA visitor program for support during an extended Summer visit in Cambridge. WNB thanks NASA ADP grant NNX11AJ59G and NASA grant NNX09AP83G for support. ACF thanks the Royal Society for support.

\section{References}

[1] Done, C., Davis, S. W., Jin, C., Blaes, O., \& Ward, M. 2012, MNRAS, 420, 1848

[2] Fabian, A. C., Miniutti, G., Gallo, L., et al. 2004, MNRAS, 353, 1071

[3] Fabian, A. C., Zoghbi, A., Ross, R. R., et al. 2009, Nature, 459, 540

[4] Fabian, A. C., Kara, E., Walton, D. J., et al. 2012, arXiv: 1208.5898

[5] Gibson R. R., Brandt W. N., Schneider D. P. 2008, ApJ, 685, 773

[6] Gierliński, M., \& Done, C. 2004, MNRAS, 349, L7

[7] Jin, C., Ward, M., Done, C., \& Gelbord, J. 2012, MNRAS, 420, 1825

[8] Just, D. W., Brandt, W. N., Shemmer, O., et al. 2007, ApJ, 665, 1004

[9] Leighly, K. M., Halpern, J. P., Helfand, D. J., Becker, R. H., \& Impey, C. D. 2001, AJ, 121, 2889

[10] Leighly, K. M., Halpern, J. P., Jenkins, E. B., et al. 2007, ApJ, 663, 103

[11] Leighly, K. M., Halpern, J. P., Jenkins, E. B., \& Casebeer, D. 2007, ApJS, 173, 1

[12] Miniutti, G., Fabian, A. C., Goyder, R., \& Lasenby, A. N. 2003, MNRAS, 344, L22

[13] Miniutti, G., \& Fabian, A. C. 2004, MNRAS, 349, 1435

[14] Miniutti, G., Fabian, A. C., Brandt, W. N., Gallo, L. C., \& Boller, T. 2009, MNRAS, 396, L85

[15] Miniutti, G., Brandt, W.N., Schneider, D.P., Fabian, A.C., Gallo, L. C., \& Boller, T. 2012, MNRAS, 425, 1718

[16] Ponti, G., Miniutti, G., Cappi, M., et al. 2006, MNRAS, 368, 903

[17] Ponti, G., Gallo, L. C., Fabian, A. C., et al. 2010, MNRAS, 406, 2591

[18] Ross, R. R., \& Fabian, A. C. 2005, MNRAS, 358, 211

[19] Wu, J., Brandt, W. N., Hall, P. B., et al. 2011, ApJ, 736, 28 